\title{
Hodge decomposition theorem for Abelian two-form gauge theory
}

\author{
E. Harikumar*, R. P. Malik ${ }^{* *, \dagger}$ and M. Sivakumar ${ }^{*, \ddagger}$ \\ * School of Physics, University of Hyderabad, \\ Hyderabad-500 046, India \\ ** S. N. Bose National Centre for Basic Sciences, \\ Block-JD, Sector-III, Salt Lake, Calcutta-700 098, India
}

\begin{abstract}
We show that the BRST/anti-BRST invariant $3+1$ dimensional 2-form gauge theory has further nilpotent symmetries (dual BRST / anti-dual BRST) that leave the gauge fixing term invariant. The generator for the dual BRST symmetry is analogous to the coexterior derivative of differential geometry. There exists a bosonic symmetry which keeps the ghost terms invariant and it turns out to be the analogue of the Laplacian operator. The Hodge duality operation is shown to correspond to a discrete symmetry in the theory. The generators of all these continuous symmetries are shown to obey the algebra of the de Rham cohomology operators of differential geometry. We derive the extended BRST algebra constituted by six conserved charges and discuss the Hodge decomposition theorem in the quantum Hilbert space of states.
\end{abstract}

\footnotetext{
$\dagger$ E-mail: malik@boson.bose.res.in

‡ E-mail: mssp@uohyd.ernet.in
} 


\section{Introduction}

For the covariant canonical quantization of gauge theories, one of the most elegant methods is the Becchi-Rouet-Stora-Tyutin (BRST) formalism [1,2] where (quantum) gauge invariance and unitarity are respected together at any arbitrary order of perturbation theory. The first-class constraints of the original gauge theories are found to be encoded in the subsidiary condition $\left(Q_{B} \mid\right.$ phys $\left.>=0\right)$ when one requires that the physical subspace (of the total Hilbert space of states) contains only those states that are annihilated by the nilpotent $\left(Q_{B}^{2}=0\right)$ and conserved $\left(\dot{Q}_{B}=0\right)$ BRST charge $Q_{B}$. In fact, the condition $Q_{B} \mid$ phys $>=0$ implies that the operator form of the first-class constraints annihilate the physical states. This requirement is essential for the consistent quantization of any theory endowed with the first-class constraints (Dirac's prescription) $[3,4]$. The nilpotency of the BRST charge $\left(Q_{B}^{2}=0\right)$ and physicality criteria $\left(Q_{B} \mid\right.$ phys $\left.>=0\right)$ are the two key requirements for the discussion of cohomological aspects of BRST formalism [5-8] and its connection with the de Rham cohomology operator $d$ (exterior derivative; $d^{2}=0$ ) of differential geometry defined on a compact manifold. For instance, two physical states are said to belong to the same cohomology class w.r.t. $Q_{B}$ if they differ by a BRST exact state as two closed forms belong to the same cohomology class w.r.t. operator $d$ if they differ by an exact form. There are two other de Rham cohomology operators that are essential for the definition of the Hodge decomposition theorem which states that, on a compact manifold, any arbitrary $n$-form $f_{n}(n=0,1,2,3 \ldots .$.$) can be written as a unique sum of a harmonic$ form $h_{n}\left(\Delta h_{n}=0, d h_{n}=0, \delta h_{n}=0\right)$, an exact form $\left(d e_{n-1}\right)$ and a co-exact form $\left(\delta c_{n+1}\right)$ :

$$
f_{n}=h_{n}+d e_{n-1}+\delta c_{n+1}
$$

where $\delta= \pm * d *,\left(\delta^{2}=0\right)$ is the dual exterior derivative, $\Delta=(d+\delta)^{2}=d \delta+\delta d$ is the Laplacian operator and $*$ is the so-called Hodge duality operation [9-12].

It is a well known fact that the cohomological operator $d$ of differential geometry finds its analogue in the local, conserved and nilpotent BRST charge $Q_{B}[7,8]$. It is, therefore, interesting to enquire if analogous local conserved charges (and corresponding local symmetry transformations for a given Lagrangian density) exist for the analogues of the other cohomological operators, viz; $\delta$ and $\Delta$. Some interesting attempts [13-16] have been made to express $\delta$ and $\Delta$ in the language of symmetry properties of a given Lagrangian density for the 1-form interacting gauge theory in any arbitrary spacetime dimension. The symmetry transformations, however, turn out to be non-local and non-covariant. In the covariant formulation [17], the nilpotency of transformations are dependent on the specific choice of parameters of the theory. Recently, it has been shown that the two-dimensional (2D) free Abelian as well as non-Abelian gauge theories (without any interaction with matter fields) provide a topological f field theoretical model for the Hodge theory where symmetry transformations corresponding to the de Rham cohomology operators $(d, \delta, \Delta)$ are nilpotent (for

\footnotetext{
* A theory with a flat spacetime metric and without any propagating degrees of freedom.
} 
$d$ and $\delta$ ), local, covariant and continuous [18-20]. The analogue of these local symmetries have also been shown to exist for the 2D topological fields (i.e., 2D Abelian gauge fields) coupled to matter (Dirac) fields in two-dimensions of spacetime [21].

In our present paper, we show the existence of symmetries corresponding to the de Rham cohomology operators for a field theoretical model in the physical four $(3+1)$ dimensional spacetime 17. The search for such symmetries in the Abelian and non-Abelian 1-form gauge theories, even though quite illuminating, has not been fully successful and satisfactory, as stated earlier. Thus, the central theme of our present work is to show that the free Abelian antisymmetric (2-form) gauge theory in 4D provides a prototype example for Hodge theory where the de Rham cohomology operators correspond to the local and conserved charges. These charges turn out to be the generators of specific local, covariant and continuous symmtery transformations for the BRST invariant Lagrangian density of this theory.

The 2-form massless gauge theory is interesting by itself as it is a dual description for the massless scalar theory. It also has interesting constraint structure: stage-one reducibility and corresponding ghost for the ghost feature. In addition, the 2-form potential also appears naturally in supergravity and superstring theories including the recent developments in non-commutative geometry [22]. Its different forms have appeared in other contexts of theoretical physics, e.g., QCD, cosmic strings and vortices, black holes, etc. [23-26]. In fact, this theory, coupled to a 1-form Abelian gauge field via a 'topological' $B \wedge F$ term, has rich mathematical structure and has been studied from various points view, viz., duality consideration [27,28], Dirac bracket analyses [29,30,31], BFT Hamiltonian formulation [32], BRST quantization [33], etc.

We shall consider the BRST invariant version (see, e.g., Section 2 (below)) of the free 4D Kalb-Ramond Lagrangian density [23,34,35]

$$
\mathcal{L}=\frac{1}{12} H^{\mu \nu \lambda} H_{\mu \nu \lambda}
$$

where $H_{\mu \nu \lambda}=\partial_{\mu} B_{\nu \lambda}+\partial_{\nu} B_{\lambda \mu}+\partial_{\lambda} B_{\mu \nu}$ is the totally antisymmetric curvature tensor constructed from the antisymmetric gauge field $B_{\mu \nu}$ f and show that: (i) in addition to the usual BRST charge $\left(Q_{B}\right)$, there exists a local, conserved and nilpotent dual(co)-BRST charge $\left(Q_{D}\right)$ under which the gauge-fixing term of this theory remains invariant. This fact should be contrasted with the usual BRST transformations, under which, it is the kinetic energy term (more precisely curvature tensor $H_{\mu \nu \lambda}$ itself) that remains invariant. (ii) The anticommutator of BRST- and dual BRST transformations leads to a symmetry transformation that is generated by a local and conserved bosonic charge $(W)$. This is analogous to the Laplacian operator in differential geometry where it is given by the anticommutator

\footnotetext{
$\dagger$ We follow the notations in which the flat Minkowski metric is $\eta_{\mu \nu}=\operatorname{diag}(+,-,-,-)$ and Levi-Civita totally antisymmetric tensor $\varepsilon_{0123}=+1=-\varepsilon^{0123}, \varepsilon_{0 i j k}=\varepsilon_{i j k}=-\varepsilon^{i j k}, \varepsilon^{\mu \nu \lambda \xi} \varepsilon_{\mu \nu \lambda \xi}=-4 !, \varepsilon^{\mu \nu \lambda \xi} \varepsilon_{\mu \nu \lambda \rho}=$ $-3 ! \delta_{\rho}^{\xi}$, etc. Here Greek indices: $\mu, \nu, \lambda \ldots \ldots=0,1,2,3$ and Latin indices: $\mathrm{i}, \mathrm{j}, \mathrm{k} \ldots \ldots . .=1,2,3$.

$\ddagger$ The gauge field $B_{\mu \nu}$ is defined through 2-form: $B=\frac{1}{2} B_{\mu \nu} d x^{\mu} \wedge d x^{\nu}$ and the curvature tensor $H_{\mu \nu \lambda}$ is defined through 3 -form as: $H=d B$. It can be readily seen that the gauge-fixing term $\partial_{\mu} B^{\mu \nu}$ can be defined through one-form by the application of $\delta$ as: $\partial_{\mu} B^{\mu \nu} d x_{\nu}=\delta B$ where $\delta=-* d *$ is the dual exterior derivative of $d$. It is clear that the gauge-fixing term is the 'Hodge dual' of curvature term.
} 
of $d$ and $\delta$. (iii) The conserved charges (e.g., $Q_{B}, Q_{D}, W$ ) can be exploited together for the discussion of the Hodge decomposition theorem in the quantum Hilbert space of states and for the anlaysis of the constraint structure on the physical (harmonic) states of the theory. (iv) A discrete transformation symmetry of the Lagrangian density relates $Q_{B}$ and $Q_{D}$ like a dual symmetry: $Q_{B} \rightarrow Q_{D}, Q_{D} \rightarrow-Q_{B}$ and $W \rightarrow-W$. This relationship maintains the anticommutator between $Q_{B}$ and $Q_{D}$ and the underlying discrete symmetry turns out to be a realization of the Hodge $*$ operation of differential geometry for this gauge theory. To the best of our knowledge, this is the first example of a field theoretical model for the Hodge theory in four $(3+1)$ dimensional spacetime where the conserved charges corresponding to the de Rham cohomology operators generate the local, continuous and covariant transformations for the fields. The existence of new symmetries (corresponding to $\delta$ and $\Delta$ ) and their generalizations might turn out to be useful in the proof of renormalizability of an interacting theory where the gauge fields are coupled to matter fields. Thus, our present work is the first step towards our main goal of having a complete understanding of the interacting theory.

The outline of our paper is as follows. In section 2, we recapitulate the essentials of the BRST formalism for the 2-form gauge theory and set up the notations for our further discussion. This is followed by the discussion and derivation of the dual BRST symmetry in section 3. We derive the symmetry generated by the Casimir operator in section 4 and obtain the corresponding conserved charge. Section 5 is devoted to the derivation of the extended BRST algebra and a brief discussion is provided for its possible connection to the de Rham cohomology operators of the differential geometry. In section 6, we discuss the Hodge decomposition theorem in the quantum Hilbert space of states and analyze the structure of constraints on the physical states of the theory. Finally, in section 7, we make some concluding remarks and point out some directions that can be pursued in the future.

\section{Preliminary: BRST symmetry}

We begin with the BRST invariant Lagrangian density [7]

$$
\begin{aligned}
\mathcal{L}_{B} & =\frac{1}{12} H^{\mu \nu \lambda} H_{\mu \nu \lambda}-\frac{1}{2} B^{\mu} B_{\mu}+B_{\nu}\left(\partial_{\mu} B^{\mu \nu}-\partial^{\nu} \phi_{1}\right)-\partial_{\mu} \bar{\beta} \partial^{\mu} \beta \\
& +\left(\partial_{\mu} \bar{C}_{\nu}-\partial_{\nu} \bar{C}_{\mu}\right) \partial^{\mu} C^{\nu}+\rho\left(\partial_{\mu} C^{\mu}+\lambda\right)+\left(\partial_{\mu} \bar{C}^{\mu}+\rho\right) \lambda
\end{aligned}
$$

where $B_{\mu}, \phi_{1}, \lambda$ and $\rho$ are the auxiliary fields 3 introduced to have the off-shell nilpotent BRST invariance. The following BRST transformations

$$
\begin{array}{lll}
\delta_{B} B_{\mu \nu}=\eta\left(\partial_{\mu} C_{\nu}-\partial_{\nu} C_{\mu}\right) & \delta_{B} C_{\mu}=\eta \partial_{\mu} \beta & \delta_{B} \bar{C}_{\mu}=\eta B_{\mu} \\
\delta_{B} \phi_{1}=-\eta \lambda & \delta_{B} \bar{\beta}=\eta \rho & \delta_{B}\left(B_{\mu}, \rho, \lambda, \beta\right)=0
\end{array}
$$

leave the Lagrangian density invariant up to a total derivative term.

\footnotetext{
$\S$ By integrating out the auxiliary fields, we will obtain the Lagrangian density which respects the on-shell nilpotent BRST symmetry.
} 
The continuous symmetry transformations (2.2) lead to the following nilpotent $\left(Q_{B}^{2}=0\right)$ and conserved $\left(\dot{Q}_{B}=0\right)$ BRST charge due to Noether theorem

$$
Q_{B}=\int d^{3} x\left[H^{0 i j} \partial_{i} C_{j}+B_{0} \lambda-\rho \dot{\beta}+\left(\partial^{0} C^{i}-\partial^{i} C^{0}\right) B_{i}-\left(\partial^{0} \bar{C}^{i}-\partial^{i} \bar{C}^{0}\right) \partial_{i} \beta\right] .
$$

This charge turns out to be the generator for the transformations (2.2) if we exploit the following general relationship

$$
\delta_{B} \Phi=-i \eta\left[\Phi, Q_{B}\right]_{ \pm}
$$

where $[,]_{ \pm}$stands for the (anti)commutator for the generic field $\Phi$ being (fermionic)bosonic in nature. For the verification of (2.4), one has to use the canonical (anti)commutators for the Lagrangian density (2.1) as given below (with $\hbar=c=1$ )

$$
\begin{array}{lc}
{\left[B_{0 i}(x, t), B^{j}(y, t)\right]=i \delta_{i}^{j} \delta(x-y)} & {[\beta(x, t), \dot{\bar{\beta}}(y, t)]=-i \delta(x-y)} \\
{\left[B_{i j}(x, t), H^{0 k l}(y, t)\right]=i\left(\delta_{i}^{k} \delta_{j}^{l}-\delta_{i}^{l} \delta_{j}^{k}\right) \delta(x-y)} \\
{\left[\phi_{1}(x, t), B_{0}(y, t)\right]=-i \delta(x-y)} & {[\bar{\beta}(x, t), \dot{\beta}(y, t)]=-i \delta(x-y)} \\
\left\{C_{0}(x, t), \rho(y, t)\right\}=-i \delta(x-y) & \left\{\bar{C}_{0}(x, t), \lambda(y, t)\right\}=i \delta(x-y) \\
\left\{C_{i}(x, t), \Pi_{C}^{j}(y, t)\right\}=i \delta_{i}^{j} \delta(x-y) & \left\{\bar{C}_{i}(x, t), \Pi_{\bar{C}}^{j}(y, t)\right\}=i \delta_{i}^{j} \delta(x-y)
\end{array}
$$

where $\delta(x-y)$ is the Dirac- delta function in 3D of space (i.e., $\left.\delta^{(3)}(\mathbf{x}-\mathbf{y})\right)$ and the expression for the canonical momenta are:

$$
\Pi_{(C)}^{(i)}=-\left(\partial^{0} \bar{C}^{i}-\partial^{i} \bar{C}^{0}\right) \quad \Pi_{(\bar{C})}^{(i)}=\left(\partial^{0} C^{i}-\partial^{i} C^{0}\right) .
$$

All the rest of the (anti)commutators are zero.

It can be readily seen that the ghost part of the Lagrangian density has the following discrete symmetry invariance

$$
\begin{array}{lll}
\beta \rightarrow \mp i \bar{\beta} & C_{\mu} \rightarrow \pm i \bar{C}_{\mu} & \rho \rightarrow \pm i \lambda \\
\bar{\beta} \rightarrow \pm i \beta & \bar{C}_{\mu} \rightarrow \pm i C_{\mu} & \lambda \rightarrow \pm i \rho .
\end{array}
$$

As a result of this symmetry, one can define an anti-BRST charge $Q_{A B}$ from (2.3) and one can obtain anti-BRST symmetry from (2.2) by exploiting (2.7). Furthermore, the total Lagrangian density (2.1) remains invariant under the following transformations:

$$
\begin{array}{lll}
B_{\mu \nu} \rightarrow B_{\mu \nu} & \phi_{1} \rightarrow \phi_{1} & B_{\mu} \rightarrow B_{\mu} \\
\beta \rightarrow e^{2 \Sigma} \beta & C_{\mu} \rightarrow e^{\Sigma} C_{\mu} & \lambda \rightarrow e^{\Sigma} \lambda \\
\bar{\beta} \rightarrow e^{-2 \Sigma} \bar{\beta} & \bar{C}_{\mu} \rightarrow e^{-\Sigma} \bar{C}_{\mu} & \rho \rightarrow e^{-\Sigma} \rho
\end{array}
$$

where $\Sigma$ is a global (spacetime independent) scale transformation parameter. This continuous symmetry leads to the derivation of a conserved ghost charge $\left(Q_{g}\right)$ as

$$
Q_{g}=\int d^{3} x\left[C_{i} \Pi_{(C)}^{(i)}+C_{0} \Pi_{(C)}^{(0)}+2 \beta \Pi_{\beta}-2 \bar{\beta} \Pi_{\bar{\beta}}-\bar{C}^{0} \Pi_{(\bar{C})}^{(0)}-\bar{C}_{i} \Pi_{(\bar{C})}^{(i)}\right]
$$


where $\Pi s$ are the canonical momenta w.r.t. ghost fields 7 . It can be readily seen, by exploiting the canonical (anti)commutators of (2.5), that

$$
\begin{aligned}
& Q_{B}^{2}=\frac{1}{2}\left\{Q_{B}, Q_{B}\right\}=0 \quad Q_{A B}^{2}=\frac{1}{2}\left\{Q_{A B}, Q_{A B}\right\}=0 \\
& \left\{Q_{B}, Q_{A B}\right\}=0 \quad i\left[Q_{g}, Q_{B}\right]=+Q_{B} \quad i\left[Q_{g}, Q_{A B}\right]=-Q_{A B}
\end{aligned}
$$

Thus, we note that $Q_{B}$ and $Q_{A B}$ are the nilpotent operators of order 2 (i.e., $Q_{B}^{2}=Q_{A B}^{2}=0$ ) and the ghost number for them is +1 and -1 respectively. This ghost number will also have relevance with some aspects of differential geometry (see, e.g., Section 5). Though the conserved and nilpotent charge $Q_{B}$ is the analogue of the exterior derivative $d[7,8]$, the conserved and nilpotent charge $Q_{A B}$ is not the analogue of the co-exterior derivative $\delta$. This is due to the fact that the anticommutator between $d$ and $\delta$ is not equal to zero (i.e., $\{d, \delta\} \neq 0)$ whereas $Q_{B}$ and $Q_{A B}$ anticommute $\left(\left\{Q_{B}, Q_{A B}\right\}=0\right)$ with each-other. Furhermore, there is no analogue of the Laplacian operator $\Delta$ in (2.10). This fact can be succinctly expressed as

$$
\begin{array}{lcc}
Q_{B}^{2}=0 & d^{2}=0 & Q_{A B}^{2}=0 \\
\left\{Q_{B}, Q_{A B}\right\}=0 & \{d, \delta\}=\Delta \neq 0
\end{array}
$$

Recently, it has been pointed out that the cohomologically higher-order BRST- and antiBRST operators do not anticommute and their anticommutator leads to the definition of a higher-order Laplacian operator for the compact non-Abelian Lie algebras [36]. This argument does not apply here in our discussion of the Abelian 2-form gauge theory because here the Lie algebra is a trivial (Abelian) algebra. Furthermore, we do not consider here the higher-order cohomology discussed in Ref. [36].

\section{Dual BRST symmetry}

In this Section, we discuss the 'dual' BRST symmetry which leaves the gauge-fixing term of the Lagrangian density invariant. This nilpotent symmetry should be contrasted with the BRST symmetry (and also anti-BRST symmetry) where it is the curvature term $H=d B$, that remains invariant. Just as one linearizes the gauge fixing term by introducing an auxiliary field $B_{\mu}$ and a scalar field $\phi_{1}$ in the case of BRST invaraint Lagrangian density (2.1), one can linearize the the kinetic energy term by incorporating another auxiliary field $\mathcal{B}_{\mu}$ and a different scalar field $\phi_{2}$ to obtain the off-shell nilpotent dual BRST invariance of the same Lagrangian density I. Such a BRST- and dual BRST invariant Lagrangian density, incorporating the above linearizations, is

$$
\begin{aligned}
\mathcal{L}_{D} & =\frac{1}{2} \mathcal{B}^{\mu} \mathcal{B}_{\mu}-\frac{1}{3 !} \varepsilon_{\mu \nu \lambda \zeta} \mathcal{B}^{\mu} H^{\nu \lambda \zeta}+\mathcal{B}^{\mu} \partial_{\mu} \phi_{2}-\frac{1}{2} B^{\mu} B_{\mu}+B_{\nu}\left(\partial_{\mu} B^{\mu \nu}-\partial^{\nu} \phi_{1}\right) \\
& -\partial_{\mu} \bar{\beta} \partial^{\mu} \beta+\left(\partial_{\mu} \bar{C}_{\nu}-\partial_{\nu} \bar{C}_{\mu}\right) \partial^{\mu} C^{\nu}+\rho\left(\partial_{\mu} C^{\mu}+\lambda\right)+\left(\partial_{\mu} \bar{C}^{\mu}+\rho\right) \lambda .
\end{aligned}
$$

I Besides (2.6), the other canonical momenta are $\Pi_{\beta}=-\dot{\bar{\beta}}, \Pi_{\bar{\beta}}=-\dot{\beta}, \Pi_{(C)}^{(0)}=-\rho, \Pi_{(\bar{C})}^{(0)}=\lambda$.

$\|$ By integrating out the linearizing field $\mathcal{B}_{\mu}$ and the scalar field $\phi_{2}$, we get back the BRST invariant Lagrangian density (2.1). 
Under the following off-shell nilpotent $\left(\delta_{D}^{2}=0\right)$ dual BRST symmetry transformations:

$$
\begin{array}{lc}
\delta_{D} B_{\mu \nu}=\eta \varepsilon_{\mu \nu \lambda \zeta} \partial^{\lambda} \bar{C}^{\zeta} \quad \delta_{D} \bar{C}_{\mu}=-\eta \partial_{\mu} \bar{\beta} & \delta_{D} C_{\mu}=\eta \mathcal{B}_{\mu} \\
\delta_{D} \beta=\eta \lambda \quad \delta_{D} \phi_{2}=-\eta \rho & \delta_{D}\left(\bar{\beta}, \lambda, \rho, \phi_{1}, B_{\mu}, \mathcal{B}_{\mu}\right)=0
\end{array}
$$

the Lagrangian density (3.1) transforms as:

$$
\delta_{D} \mathcal{L}_{D}=-\eta \partial_{\mu}\left[\rho \mathcal{B}^{\mu}+\lambda \partial^{\mu} \bar{\beta}+\left(\partial^{\mu} \bar{C}^{\nu}-\partial^{\nu} \bar{C}^{\mu}\right) \mathcal{B}_{\nu}\right]
$$

Thus, the above Lagrangian density (3.1) remains invariant under the dual BRST transformations (3.2) and the BRST transformations (2.2) (together with $\delta_{B}\left(\mathcal{B}_{\mu}, \phi_{2}\right)=0$ ). It is appropriate to call the symmetry transformations (3.2) as the 'dual' BRST transformations because it is the gauge-fixing term (i.e., $\delta B=\partial_{\mu} B^{\mu \nu} d x_{\nu}$ : the Hodge dual of the curvature $d B=H$ ) of the theory that remains invariant and the kinetic energy term (which remains invariant under BRST- and anti-BRST symmetries) transforms under it to compensate for the transformation of the ghost terms. The Noether conserved current, derived from the above symmetry transformations, is:

$$
J_{D}^{\alpha}=\varepsilon^{\alpha \beta \rho \sigma} B_{\beta} \partial_{\rho} \bar{C}_{\sigma}-\mathcal{B}^{\alpha} \rho-\lambda \partial^{\alpha} \bar{\beta}-\left(\partial^{\alpha} C^{\lambda}-\partial^{\lambda} C^{\alpha}\right) \partial_{\lambda} \bar{\beta}-\left(\partial^{\alpha} \bar{C}^{\lambda}-\partial^{\lambda} \bar{C}^{\alpha}\right) \mathcal{B}_{\lambda}
$$

which ultimately leads to the derivation of a conserved $\left(\dot{Q}_{D}=0\right)$ and nilpotent $\left(Q_{D}^{2}=0\right)$ dual BRST charge $\left(Q_{D}=\int d^{3} x J_{D}^{0}\right)$ as:

$$
Q_{D}=\int d^{3} x\left[\varepsilon^{0 i j k}\left(B_{i}\right) \partial_{j} \bar{C}_{k}-\mathcal{B}_{0} \rho-\lambda \dot{\bar{\beta}}-\left(\partial^{0} C^{i}-\partial^{i} C^{0}\right) \partial_{i} \bar{\beta}-\left(\partial^{0} \bar{C}^{i}-\partial^{i} \bar{C}^{0}\right) \mathcal{B}_{i}\right] .
$$

To prove the conservation law for the Noether current in (3.4), one has to use some of the following equations of motion derived from the Lagrangian density (3.1)

$$
\begin{aligned}
& \partial \cdot B=0 \quad \partial \cdot \mathcal{B}=0 \quad \square \phi_{1}=\square \phi_{2}=0 \quad B_{\mu}=\partial^{\rho} B_{\rho \mu}-\partial_{\mu} \phi_{1} \\
& \mathcal{B}_{\mu}=\frac{1}{3 !} \varepsilon_{\mu \nu \lambda \xi} H^{\nu \lambda \xi}-\partial_{\mu} \phi_{2} \quad \square \rho=\square \lambda=\square \beta=\square \bar{\beta}=0 \\
& \square C^{\mu}-\partial^{\mu}(\partial \cdot C)+\partial^{\mu} \lambda=0 \quad \partial_{\mu} C^{\mu}+2 \lambda=0 \text {, } \\
& \square \bar{C}^{\mu}-\partial^{\mu}(\partial \cdot \bar{C})+\partial^{\mu} \rho=0 \quad \partial_{\mu} \bar{C}^{\mu}+2 \rho=0 \\
& \square B_{\mu}-\partial_{\mu}(\partial \cdot B)=0 \rightarrow \square B_{\mu}=0 \\
& \square \mathcal{B}_{\mu}-\partial_{\mu}(\partial \cdot \mathcal{B})=0 \rightarrow \square \mathcal{B}_{\mu}=0 \\
& \varepsilon_{\mu \nu \lambda \xi} \partial^{\lambda} \mathcal{B}^{\xi}+\left(\partial_{\mu} B_{\nu}-\partial_{\nu} B_{\mu}\right)=0 \text {. }
\end{aligned}
$$

As the ghost part of the Lagrangian density (3.1) remains invariant under (2.7), it is very interesting to note that the bosonic part of this Lagrangian density remains invariant under the following discrete symmetry transformations

$$
\begin{array}{ll}
\mathcal{B}_{\mu} \rightarrow \mp i B_{\mu} & \phi_{2} \rightarrow \mp i \phi_{1} \quad \phi_{1} \rightarrow \pm i \phi_{2} \\
B_{\mu} \rightarrow \pm i \mathcal{B}_{\mu} & B_{\mu \nu} \rightarrow \mp \frac{i}{2} \varepsilon_{\mu \nu \lambda \xi} B^{\lambda \xi} .
\end{array}
$$

It is straightforward to check that that the total Lagrangian density (3.1) remains invariant under the combination of discrete symmetry transformations (2.7) and (3.7). We note here that the analogue of Hodge $*$ operation of differential geometry turns out to be the combined 
symmetries (2.7) and (3.7). This assertion can be verified by the validity of the following relation

$$
\delta_{D}(\Phi)= \pm * \delta_{B} *(\Phi)
$$

where $(+)$ - stands for the generic field $\Phi$ being (bosonic) fermionic in nature, $\delta_{D}$ and $\delta_{B}$ are the nilpotent transformations (2.2) and (3.2) and $*$ operation is the discrete transformations (2.7) and (3.7). Thus, we note that the dual BRST and BRST variations (on a field) are related to each-other in the same way as the action of an exterior derivative $d$ and coexterior derivative $\delta= \pm * d *$ on a given differential form. This symmetry is also reflected in the expressions for BRST- and dual BRST charges. In fact, it can be readily seen that under the transformations (2.7) and (3.7), one obtains the following changes for these conserved and nilpotent charges:

$$
Q_{B} \rightarrow Q_{D} \quad Q_{D} \rightarrow-Q_{B}
$$

In the language of symmetry transformations, this fact can be translated into: $\delta_{B}(\Phi) \rightarrow$ $\delta_{D}(\Phi), \quad \delta_{D}(\Phi) \rightarrow-\delta_{B}(\Phi)$ under (2.7) and (3.7). Here $\Phi$ is the generic field representing bosonic as well as fermionic variables of the theory. It is interesting to note the similarity between relations (3.9) and the usual electro-magnetic duality present in the case of Maxwell equations (for $U(1)$ gauge theory) where $\mathbf{E} \rightarrow \mathbf{B}, \quad \mathbf{B} \rightarrow-\mathbf{E}$ under global duality transformations (see, e.g., Ref. [37,38]).

The existence of discrete symmetry for the ghost action, allows one to define an anti-dual BRST charge $Q_{A D}$ from the expression for $Q_{D}$ in (3.5). The off-shell nilpotent transformations generated by $Q_{A D}$ can be also derived from (3.2) by exploiting (2.7). Now, it is evident that the total Lagrangian density (3.1) respects four nilpotent symmetries which are generated by (anti) BRST- and (anti) dual BRST charges. The exact expressions for these charges for the Lagrangian density (3.1) are

$$
\begin{aligned}
& Q_{B}=\int d^{3} x\left[\varepsilon^{0 i j k} \mathcal{B}_{i} \partial_{j} C_{k}+B_{0} \lambda-\rho \dot{\beta}+\left(\partial^{0} C^{i}-\partial^{i} C^{0}\right) B_{i}-\left(\partial^{0} \bar{C}^{i}-\partial^{i} \bar{C}^{0}\right) \partial_{i} \beta\right] \\
& Q_{D}=\int d^{3} x\left[\varepsilon^{0 i j k} B_{i} \partial_{j} \bar{C}_{k}-\mathcal{B}_{0} \rho-\lambda \dot{\bar{\beta}}-\left(\partial^{0} C^{i}-\partial^{i} C^{0}\right) \partial_{i} \bar{\beta}-\left(\partial^{0} \bar{C}^{i}-\partial^{i} \bar{C}^{0}\right) \mathcal{B}_{i}\right] \\
& Q_{A B}=i \int d^{3} x\left[\varepsilon^{0 i j k} \mathcal{B}_{i} \partial_{j} \bar{C}_{k}+B_{0} \rho+i \lambda \dot{\bar{\beta}}+\left(\partial^{0} \bar{C}^{i}-\partial^{i} \bar{C}^{0}\right) B_{i}+i\left(\partial^{0} C^{i}-\partial^{i} C^{0}\right) \partial_{i} \bar{\beta}\right] \\
& Q_{A D}=i \int d^{3} x\left[\varepsilon^{0 i j k} B_{i} \partial_{j} C_{k}-\mathcal{B}_{0} \lambda-i \rho \dot{\beta}-\left(\partial^{0} C^{i}-\partial^{i} C^{0}\right) \mathcal{B}_{i}-i\left(\partial^{0} \bar{C}^{i}-\partial^{i} \bar{C}^{0}\right) \partial_{i} \beta\right] .
\end{aligned}
$$




\section{Bosonic symmetry}

It is evident that the total Lagrangian density $\mathcal{L}_{D}$ in (3.1) is endowed with four nilpotent symmetry transformations that are generated by the conserved and nilpotent charges (3.10-3.13). It is logical to expect that the anticommutator of the pair of these symmetries would also be the symmetry for (3.1). Since four anticommutators $\left(\left\{Q_{B}, Q_{A B}\right\}=\right.$ $\left.0,\left\{Q_{D}, Q_{A D}\right\}=0,\left\{Q_{B}, Q_{A D}\right\}=0,\left\{Q_{D}, Q_{A B}\right\}=0\right)$ are zero, the other two anticommutators $\left(\left\{Q_{B}, Q_{D}\right\},\left\{Q_{A B}, Q_{A D}\right\}\right)$ would lead to the definition of a bosonic operator $W$ which will generate a symmetry transformation $\delta_{W}$ for (3.1). The following transformations generated by the operator $W$ (with $\left.\kappa=-i \eta \eta^{\prime}\right)$

$$
\begin{aligned}
& \delta_{W} B_{\mu \nu}=i \kappa\left(\partial_{\mu} \mathcal{B}_{\nu}-\partial_{\nu} \mathcal{B}_{\mu}+\varepsilon_{\mu \nu \lambda \xi} \partial^{\lambda} B^{\xi}\right) \quad \delta_{W} \phi_{1}=0 \quad \delta_{W} \phi_{2}=0, \delta_{W} B_{\mu}=0 \\
& \delta_{W} C_{\mu}=i \kappa \partial_{\mu} \lambda \quad \delta_{W} \bar{C}_{\mu}=-i \kappa \partial_{\mu} \rho \quad \delta_{W} \rho=0, \quad \delta_{W} \lambda=0 \quad \delta_{W} \mathcal{B}_{\mu}=0 \\
& \delta_{W}(\partial \cdot C)=i \kappa \square \lambda \quad \delta_{W}(\partial \cdot \bar{C})=-i \kappa \square \rho \quad \delta_{W}\left(\partial^{\rho} B_{\rho \mu}\right)=i \kappa\left(\square \mathcal{B}_{\mu}-\partial_{\mu}(\partial \cdot \mathcal{B})\right) \\
& \delta_{W}\left(\frac{1}{3 !} \varepsilon_{\mu \nu \lambda \xi} H^{\nu \lambda \xi}\right) \equiv \delta_{W}\left(\frac{1}{2} \varepsilon_{\mu \nu \lambda \xi} \partial^{\nu} B^{\lambda \xi}\right)=i \kappa\left(\square B_{\mu}-\partial_{\mu}(\partial \cdot B)\right) \delta_{W} \beta=0 \delta_{W} \bar{\beta}=0
\end{aligned}
$$

turn out to be the symmetry transformations for $\mathcal{L}_{D}$;

$$
\begin{aligned}
\delta_{W} \mathcal{L}_{D} & =i \kappa \partial_{\alpha}\left[X^{\alpha}\right] \\
X^{\alpha} & =\rho \partial^{\alpha} \lambda-\partial^{\alpha} \rho \lambda+B^{\rho} \partial^{\alpha} \mathcal{B}_{\rho} \\
& -\mathcal{B}^{\rho} \partial^{\alpha} B_{\rho}+\mathcal{B}^{\alpha}(\partial \cdot B)-B^{\alpha}(\partial \cdot \mathcal{B}) .
\end{aligned}
$$

Here $\eta$ and $\eta^{\prime}$ (in the definition of $\kappa$ ) are the fermionic spacetime indpendent parameters in the transformations corresponding to $\delta_{B}$ and $\delta_{D}$ of eqns. (2.2) and (3.2). The Noether conserved current corresponding to the transformations (4.1), is

$$
\begin{aligned}
J_{W}^{\alpha} & =i \varepsilon^{\alpha \beta \rho \sigma}\left(\mathcal{B}_{\beta} \partial_{\rho} \mathcal{B}_{\sigma}+B_{\beta} \partial_{\rho} B_{\sigma}\right)+i \partial_{\rho}\left(\mathcal{B}^{\rho} B^{\alpha}-\mathcal{B}^{\alpha} B^{\rho}\right) \\
& +i\left(\partial^{\alpha} C^{\lambda}-\partial^{\lambda} C^{\alpha}\right) \partial_{\lambda} \rho+i\left(\partial^{\alpha} \bar{C}^{\mu}-\partial^{\mu} \bar{C}^{\alpha}\right) \partial_{\mu} \lambda
\end{aligned}
$$

which finally leads to the derivation of a local conserved charge $\left(W=\int d^{3} x J_{W}^{0}\right)$ as

$$
W=i \int d^{3} x\left[\varepsilon^{0 i j k}\left(B_{i} \partial_{j} B_{k}+\mathcal{B}_{i} \partial_{j} \mathcal{B}_{k}\right)+\left(\partial^{0} C^{i}-\partial^{i} C^{0}\right) \partial_{i} \rho+\left(\partial^{0} \bar{C}^{i}-\partial^{i} \bar{C}^{0}\right) \partial_{i} \lambda\right] .
$$

This charge can be directly computed from the anticommutators of $\left\{Q_{B}, Q_{D}\right\}$ or $\left\{Q_{A B}, Q_{A D}\right\}$ by exploiting the analogue of canonical (anti)commutators in (2.5) for the Lagrangian density (3.1). In fact, all the (anti)commuators of (2.5) remain intact except the fact that now the canonical momenta w.r.t. $B_{k l}$ becomes $\varepsilon^{0 k l m} \mathcal{B}_{m}$ instead of $H^{0 k l}$. Thus, one has to replace now $H^{0 k l}$ by $\varepsilon^{0 k l m} \mathcal{B}_{m}$ in one of the commutators of eqn. (2.5).

There are simpler ways to compute this generator $W$ for the bosonic symmetry transformations in (4.1). Since the conserved and nilpotent charges in (3.10-3.13) are the generators of the nilpotent transformations, it can be readily seen that the following equations

$$
\begin{aligned}
& \delta_{B} Q_{D}=-i \eta\left\{Q_{D}, Q_{B}\right\}=-i \eta W \\
& \delta_{D} Q_{B}=-i \eta\left\{Q_{B}, Q_{D}\right\}=-i \eta W \\
& \delta_{A B} Q_{A D}=-i \eta\left\{Q_{A D}, Q_{A B}\right\}=-i \eta W \\
& \delta_{A D} Q_{A B}=-i \eta\left\{Q_{A B}, Q_{A D}\right\}=-i \eta W
\end{aligned}
$$


can be exploited to derive $W$ from the expressions of charges in (3.10-3.13) and the transformations (2.2) and (3.2). It will be noticed that here $\delta_{A B}$ and $\delta_{A D}$ correspond to anti-BRSTand anti-dual BRST transformations that can be easily derived from eqns. (2.2) and (3.2). It is straightforward to check that $\delta_{D} Q_{B}=-i \eta W$ leads to:

$$
W=i \int d^{3} x\left[\varepsilon^{0 i j k} \mathcal{B}_{i} \partial_{j} \mathcal{B}_{k}+\rho \dot{\lambda}+\left(\partial^{0} \mathcal{B}^{i}-\partial^{i} \mathcal{B}^{0}\right) B_{i}+\left(\partial^{0} \bar{C}^{i}-\partial^{i} \bar{C}^{0}\right) \partial_{i} \lambda\right]
$$

We can also obtain an expression for $W$ from the expression for $Q_{D}$ by applying the transformations $\delta_{B}$ (i.e., $\left.\delta_{B} Q_{D}=-i \eta W\right)$ as given below

$$
W=i \int d^{3} x\left[\varepsilon^{0 i j k} B_{i} \partial_{j} B_{k}+\lambda \dot{\rho}-\left(\partial^{0} B^{i}-\partial^{i} B^{0}\right) \mathcal{B}_{i}+\left(\partial^{0} C^{i}-\partial^{i} C^{0}\right) \partial_{i} \rho\right] .
$$

It is obvious that the expressions (4.6) and (4.7) bear a different outlook than the expression derived in (4.4). All these expressions for $W$ are, however, identical if we exploit the appropriate equations of motion. Similar expressions emerge from the calculations of other expressions in (4.5). The most concise form of $W$ that can be derived from (4.5), is

$$
W=i \int d^{3} x\left[\varepsilon^{0 i j k}\left(B_{i} \partial_{j} B_{k}+\mathcal{B}_{i} \partial_{j} \mathcal{B}_{k}\right)+\lambda \dot{\rho}+\rho \dot{\lambda}\right] .
$$

It will be noticed that we have exploited here only the off-shell nilpotent symmetries (and conserved charges) for the derivation of $W$.

One important point to be noticed here is the fact that the operator $W$ does not go to zero if we exploit the equations of motion. This feature is completely different from the discussion of the free 2D (non)Abelian gauge theories in Refs. [18-20] where it has been argued that the topological nature of these theories is encoded in the vanishing of the operator $W$ when equations of motion are used and all the fields are assumed to fall off rapidly at infinity.

\section{Extended BRST algebra}

In this Section, we concentrate on the derivation of an extended BRST algebra (which is found to be constituted by six conserved charges) and provide a possible connection of this algebra with the algebra of the de Rham cohomology operators of differential geometry. In the normal BRST algebra, there are three conserved charges (viz., $Q_{g}, Q_{B}, Q_{A B}$ ) of equations (2.9), (3.10) and (3.12). The existence of new symmetries, however, provide three more conserved charges (viz., $Q_{D}, Q_{A D}$ and $W$ ) which are given by equations (3.11), (3.13) and (4.8). If one exploits the canonical (anti)commutators of equation (2.5) for the Lagrangian density (3.1), one can show that all the six conserved charges obey the following extended BRST algebra

$$
\begin{aligned}
& {\left[W, Q_{k}\right]=0 \quad k=g, B, A B, D, A D} \\
& Q_{B}^{2}=0 \quad Q_{D}^{2}=0 \quad Q_{A B}^{2}=0 \quad Q_{A D}^{2}=0 \\
& i\left[Q_{g}, Q_{B}\right]=+Q_{B} \quad i\left[Q_{g}, Q_{D}\right]=-Q_{D} \quad i\left[Q_{g}, Q_{A B}\right]=-Q_{A B} \\
& i\left[Q_{g}, Q_{A D}\right]=+Q_{A D} \quad\left\{Q_{B}, Q_{A B}\right\}=0 \quad\left\{Q_{D}, Q_{A D}\right\}=0 \\
& \left\{Q_{B}, Q_{A D}\right\}=0 \quad\left\{Q_{D}, Q_{A B}\right\}=0 \quad\left\{Q_{B}, Q_{D}\right\}=\left\{Q_{A B}, Q_{A D}\right\}=W .
\end{aligned}
$$


A few comments are in order. First of all, it is trivial to see that the operator $W$ is the Casimir operator for the whole extended BRST algebra. Secondly, there are four nilpotent (of order two) charges in the extended BRST algebra. Thirdly, two anticommutators (viz. $\left.\left\{Q_{B}, Q_{D}\right\},\left\{Q_{A B}, Q_{A D}\right\}\right)$ lead to the definition of of the Casimir operator $W$. And, lastly, the ghost number for charges $Q_{B}$ and $Q_{A D}$ is +1 and that of $Q_{D}$ and $Q_{A B}$ is -1 . There are simpler ways to check the validity of the above statements. For instance, exploiting the symmetry transformations of eqns. (2.2), (3.2), (4.1) and an infinitesimal version of (2.8), it can be easily seen that

$$
\begin{array}{lcc}
\delta_{B} W=0 & \delta_{D} W=0 & \delta_{g} W=0 \\
\delta_{A B} W=0 & \delta_{A D} W=0 & \delta_{W} W=0
\end{array}
$$

where the expression for the $W$ operator can be taken to be its most concise form of eqn. (4.8). Similarly other expressions for the (anti)commutators in (5.1) can be checked by merely using the symmetry transformation properties and the expressions for the conserved charges.

Next we present arguments to bring out the analogy between symmetry generators of this field theoretical model and the de Rham cohomology operators. It is a well known fact that the de Rham cohomology operators $(d, \delta, \Delta)$ obey the following algebra

$$
\begin{array}{lcc}
d^{2}=0 & \delta^{2}=0 & \Delta=(d+\delta)^{2}=d \delta+\delta d \\
{[\Delta, d]=0} & {[\Delta, \delta]=0} & \Delta=\{d, \delta\} .
\end{array}
$$

Furthermore, a differential form of degree $n\left(f_{n}\right)$ becomes a differential form of degree $n+1$ $\left(f_{n+1}\right)$ due to the application of operator $d$ (i.e., $\left.d f_{n} \sim f_{n+1}\right)$. In contrast, the operator $\delta$ reduces the degree of a form by one (i.e., $\delta f_{n} \sim f_{n-1}$ ) on which it acts and the Laplacian operator $\Delta$ does not change the degree of the form (i.e., $\Delta f_{n} \sim f_{n}$ ). Now we observe that the ghost number of the state is parallel to the degree of the differential form and $Q_{B}, Q_{D}$ and $W$ play respectively the role of $d, \delta$ and $\Delta$ in differential geometry. Exploiting the algebra (5.1), it can be readily seen that a state $\mid \psi>_{n}$ with ghost number $n$ (i.e., $\left.i Q_{g}\left|\psi>_{n}=n\right| \psi>_{n}\right)$ in the quantum Hilbert space will imply that the ghost number for the states $Q_{B}\left|\psi>_{n}, Q_{D}\right| \psi>_{n}, W \mid \psi>_{n}$ is $(n+1),(n-1), n$ respectively. This fact can be succinctly expressed as

$$
\begin{aligned}
i Q_{g} Q_{B} \mid \psi>_{n} & =(n+1) Q_{B} \mid \psi>_{n} \\
i Q_{g} Q_{D} \mid \psi>_{n} & =(n-1) Q_{D} \mid \psi>_{n} \\
i Q_{g} W \mid \psi>_{n} & =n W \mid \psi>_{n} \\
i Q_{g} Q_{A B} \mid \psi>_{n} & =(n-1) Q_{A B} \mid \psi>_{n} \\
i Q_{g} Q_{A D} \mid \psi>_{n} & =(n+1) Q_{A D} \mid \psi>_{n} .
\end{aligned}
$$

Thus, now one can draw a parallel between the differential geometry (and the corresponding de Rham cohomology operators) defined on a compact manifold and the quantum states, conserved charges, etc., defined in the quantum Hilbert space of states. For instance, the differential forms are just like quantum states; a closed form $(d f=0)$ is just like a BRST 
closed (physical) state $\left(Q_{B} \mid \psi>=0\right.$ ); a compact manifold is just like the quantum Hilbert space of states; degree of a form is analogous to the ghost number and the de Rham cohomolgy operators $(d, \delta, \Delta)$ have their counterpart as conserved charges $\left(Q_{B}, Q_{D}, W\right)$ and $\left(Q_{A D}, Q_{A B}, W\right)$, etc. It is a very special feature of the BRST formalism that each of the de Rham cohomology operators $d, \delta$ can be identified with two symmetry generators. This, in turn, implies that irrespective of the nature (i.e., real or complex) of the compact manifold, its counterpart - - the quantum Hilbert space of states - - is always complex so that $d$ and $\delta$ have two representations and the analogue of the Laplacian operator (i.e., $W$ ) can also be expressed in two different ways (i.e., $\left\{Q_{B}, Q_{D}\right\}=\left\{Q_{A B}, Q_{A D}\right\}=W$ ). However, if we retrace back, the full strength of the BRST cohomology and Hodge decomposition theorem implies that the compact manifold has to be a complex manifold so that one can achieve a complete analogy with BRST formalism. In other words, it should be possible to define $(d, \bar{d}),(\delta, \bar{\delta})$ and $(\Delta, \bar{\Delta})$ on the compact manifold so that cohomology operators $\Delta=\bar{\Delta}$ and $\Delta=d \delta+\delta d \equiv \bar{d} \bar{\delta}+\bar{\delta} \bar{d}$ can be constructed on this manifold.

\section{Constraint analysis}

In this Section, we first discuss the Hodge decomposition theorem for a given state $\mid \psi>_{n}$ (with ghost number $n$ ) in the quantum Hilbert space of states. This is, then, followed by the discussion of constraints on the physical (harmonic) states by the imposition of the physicality criteria with conserved and nilpotent charges (i.e., $Q_{B} \mid$ phys $>=0, Q_{D} \mid$ phys $>=$ 0 ) which define the physical subspace of states in the total quantum Hilbert space of states. It is obvious from the algebra (5.1) and the ghost number analysis in eqn. (5.4) that, now any arbitrary state $\mid \psi>_{n}$ in the quantum Hilbert space of states can be written as

$$
\left|\psi>_{n}=\right| \omega>_{n}+Q_{B}\left|\theta>_{n-1}+Q_{D}\right| \chi>_{n+1}
$$

where $\mid \omega>_{n}$ is the harmonic state (i.e., $\left.W\left|\omega>_{n}=0, Q_{B}\right| \omega>_{n}=0, Q_{D} \mid \omega>_{n}=0\right), Q_{B} \mid \theta>_{n-1}$ is a BRST exact state and $Q_{D} \mid \chi>_{n+1}$ is a co-BRST exact state. This equation is just the analogue of the Hodge decomposition theorem (1.1) written for a differential form in terms of the de Rham cohomology operators $(d, \delta, \Delta)$ defined on a compact manifold. It is a special feature of the BRST formalism (and the corresponding extended BRST algebra (5.1)) that eqn. (1.1) can also be expressed in terms of the conserved and nilpotent charges $Q_{A B}$ and $Q_{A D}$ as

$$
\left|\psi>_{n}=\right| \omega>_{n}+Q_{A D}\left|\theta>_{n-1}+Q_{A B}\right| \chi>_{n+1}
$$

where $Q_{A B}$ and $Q_{A D}$ are the anti-BRST and anti-dual BRST charges*.

\footnotetext{
* Unlike the uniqueness of the Hodge decomposition in the mathematical aspects of the de Rham cohomology, the uniqueness of the corresponding decomposition of the quantum states (cf. eqns. $(6.1,6.2))$ is not obvious in the quantum Hilbert space of states.
} 
It is a noteworthy point that the combined discrete transformations (2.7) and (3.7), turn out to be the symmetry of the Lagrangian density of the theory under discussion. It is obvious from our earlier arguments that this symmetry corresponds to the Hodge duality operation (i.e., * operation) in differential geometry. Thus, we have a theory which is duality invariant due to the presence of the discrete symmetries (2.7) and (3.7). As a result, the vacuum- and physical states of the quantum theory should be also duality (i.e., BRST and dual BRST) invariant in the quantum Hilbert space of states. This feature, in fact, has been exploited in Refs. [18-20] to establish the topological nature of the 2D free (non)Abelian gauge theory. In the BRST formalism, physical states are those states that are annihilated by $Q_{B}$ (i.e. $Q_{B} \mid$ phys $>=0$ ). Due to the presence of the discrete symmetry, it is obvious that $Q_{B}$ goes to $Q_{D}$ (cf. (3.9)) and hence the latter also annihilate the physical states (i.e., $Q_{B} \mid$ phys $>=0 \rightarrow Q_{D} \mid$ phys $>=0$ ). These two together imply that the Casimir operator $W$ also annhilates the physical states. It is, therefore, clear that the physical states are the harmonic states. Of course, the vacuum state will be annihilated by all these charges, as they are the generators of the unbroken symmetry transformations**. Thus, these states satisfy

$$
\begin{array}{lcc}
W \mid \text { vac }>=0 & Q_{B} \mid \text { vac }>=0 & Q_{D} \mid \text { vac }>=0 \\
Q_{A B} \mid \text { vac }>=0 & Q_{A D} \mid \text { vac }>=0 \\
W \mid \text { phys }>=0 & Q_{B} \mid \text { phys }>=0 & Q_{D} \mid \text { phys }>=0 \\
Q_{A B} \mid \text { phys }>=0 & Q_{A D} \mid \text { phys }>=0 .
\end{array}
$$

The conditions $i Q_{g} \mid$ phys $>=0$ and $i Q_{g} \mid$ vac $>=0$ imply that the ghost number of the physical- and vacuum states is zero. No other constraint emerge on physical states due to the existence of ghost charge $Q_{g}$. It will be noted that the conditions $\left(Q_{B} \mid\right.$ phys $>=$ $0, Q_{A B} \mid$ phys $>=0$ ) lead to the one and the same constraints on the physical state. Thus, we can choose one of them for the constraint analysis. Similar argument holds for the conditions $Q_{D} \mid$ phys $>=0$ and $Q_{A D} \mid$ phys $>=0$ and one can choose only one of these charges for the discussion of constraints ${ }^{\ddagger}$. Thus, we see that the vacuum, as well as the physical (harmonic) states, of the theory respect three basic symmetries (cf. (6.3)) and the ghost number for them is zero. It will be noticed that these conclusions are arrived at by the symmetry considerations alone.

Before we concentrate on the constraint analysis of the Lagrangian density in (3.1), we shall dwell a bit on the nature of constraints for the original Lagrangian density $\mathcal{L}$ of eqn. (1.2). It is evident that the canonical momenta w.r.t. the antisymmetric field $B_{\mu \nu}$ is:

\footnotetext{
** If the discrete transformations (2.7) and (3.7) (which relate $Q_{B}$ and $Q_{D}$ ) are not the symmetry of the Lagrangian density, the physical (harmonic) states can be assumed to be annihilated independently by the BRST and the dual BRST charges.

$\ddagger$ In what follows, we shall concentrate on the set of operators $Q_{B}, Q_{D}, W$ for the discussion of the Hodge decomposition theorem as well as the constraint analysis. However, our arguments and analysis will be valid for the set of operators: $Q_{A B}, Q_{A D}, W$ as well.
} 
$\Pi^{\mu \nu}=H^{0 \mu \nu}$ and the equations of motion are: $\partial_{\mu} H^{\mu \nu \lambda}=0$. Thus, it is clear that $\Pi^{0 i} \approx 0$ is the primary constraint and the secondary constraint is nothing but the equation of motion w.r.t. $B_{0 i}$ field, i.e., $\partial_{j} H^{o i j} \equiv \partial_{j} \Pi^{i j} \approx 0$. Both these constraints are first-class $[28,29]$ in the language of Dirac and they imply the existence of a gauge symmetry in the theory. For the consistent quantization of this theory, it is essential that $\Pi^{0 i} \mid$ phys $>=0, \partial_{j} \Pi^{i j} \mid$ phys $>=0$ (Dirac's presecription). We shall see that exactly these constraints will appear when we shall demand: $Q_{B} \mid$ phys $>=0$ (for the Lagrangian density (3.1)). Its dual description will emerge from the requirement: $Q_{D} \mid$ phys $>=0$.

It can be readily seen that the requirement $Q_{B} \mid$ phys $>=0$, for the Lagrangian density (3.1), leads to the following constraints on the theory:

$$
\begin{array}{lll}
\Pi^{0 i}\left(=B^{i}\right) \mid \text { phys }>=0 & \rightarrow & \left(\partial_{\rho} B^{\rho i}-\partial^{i} \phi_{1}\right) \mid \text { phys }>=0 \\
\partial_{j} \Pi^{i j}\left(=\partial_{0} B^{i}\right) \mid \text { phys }>=0 & \rightarrow & \left(-\varepsilon^{o i j k} \partial_{j} \mathcal{B}_{k}\right) \mid \text { phys }>=0
\end{array}
$$

where the expression for $Q_{B}$ has been taken from eqn. (3.10) and equations of motion from (3.6) have been used for the above derivation. Furthermore, it has been assumed here that the ghost fields, present in the expression for $Q_{B}$, do not lead to any constraints on the physical states of the theory. It is evident that in the above equation, we retrieve the constraints of the original gauge theory described by the Lagrangian density (1.2). Now the requirement $Q_{D} \mid$ phys $>=0$ leads to

$$
\begin{array}{lll}
\left(\mathcal{B}^{i}\right) \mid \text { phys }>=0 & \rightarrow & \left(\frac{1}{2} \varepsilon^{i \nu \lambda \xi} \partial_{\nu} B_{\lambda \xi}-\partial^{i} \phi_{2}\right) \mid \text { phys }>=0 \\
\left(\partial_{0} \mathcal{B}^{i}\right) \mid \text { phys }>=0 & \rightarrow \quad\left(+\varepsilon^{o i j k} \partial_{j} B_{k}\right) \mid \text { phys }>=0 .
\end{array}
$$

Exploiting eqn. (3.7) of the duality transformations for the bosonic part of the Lagrangian density, it can be checked that the above constraints in (6.5) are just the 'dual description' of the constraints obtained in (6.4), though they appear different.

It will be noticed that even though the auxiliary field $B_{0}$ is present in the expression for $Q_{B}$, we have not written $Q_{B} \mid$ phys $>=0$ implies $B_{0} \mid$ phys $>=0$. This is because of the fact that $B_{0}$ is a conserved quantity and it remains the same w.r.t. time evolution. In fact, it can be easily seen that the quantity: $I_{0}=\int d^{3} x B_{0}$ is a time evolution invariant operator due to equations of motion in (3.6) (i.e., $\partial_{0} B_{0}=\partial_{i} B_{i}$ ). Thus, $B_{0} \mid$ phys $>=0$ is a trivial constraint on the theory. Similarly, we have not concluded from the restriction $Q_{D} \mid$ phys $>=0$, the obvious constraint $\mathcal{B}_{0} \mid$ phys $>=0$ as there is no evolution for the $\mathcal{B}_{0}$ field due to $\partial \cdot \mathcal{B}=0$ (cf. (3.6)). Strictly speaking, however, these constraints should be incorporated in (6.4) and (6.5) respectively. In fact, these finally imply that $\Pi_{\phi_{1}}\left(=-B_{0}\right) \mid$ phys $>=0$ and $\Pi_{\phi_{2}}\left(=\mathcal{B}_{0}\right) \mid$ phys $>=0$. More precisely, the constraints $B_{0} \mid$ phys $>=0, B_{i} \mid$ phys $>=0$ and its counterpart $\mathcal{B}_{0} \mid$ phys $>=0, \mathcal{B}_{i} \mid$ phys $>=0$ together imply that:

$$
\begin{array}{lll}
\left(\mathcal{B}_{\mu}\right) \mid \text { phys }>=0 & \rightarrow & \left(\frac{1}{2} \varepsilon^{\mu \nu \lambda \xi} \partial_{\nu} B_{\lambda \xi}-\partial^{\mu} \phi_{2}\right) \mid \text { phys }>=0 \\
\left(B_{\mu}\right) \mid \text { phys }>=0 & \rightarrow \quad\left(\partial_{\rho} B^{\rho \mu}-\partial^{\mu} \phi_{1}\right) \mid \text { phys }>=0 .
\end{array}
$$

This shows that the total gauge-fixing term $\left(\partial^{\rho} B_{\rho \mu}-\partial_{\mu} \phi_{1}\right)$ and its dual annihilate the physical states of the theory. These conditions gauge away some of the degrees of freedom 
of the $B_{\mu \nu}$ gauge field. It is straightforward to see that the constraints $W \mid$ phys $>=0$ does not lead to any new restrictions on the physical state. In fact, it encompasses both the constraints given in eqns. (6.4) and (6.5) due to $Q_{B} \mid$ phys $>=0$ and $Q_{D} \mid$ phys $>=0$. This is due to the fact that $W=\left\{Q_{B}, Q_{D}\right\}$ and $W \mid$ phys $>=0$ implies that $Q_{B} \mid$ phys $>=0$ and $Q_{D} \mid$ phys $>=0$ which are in some sense, unique solutions to the constraint $W \mid$ phys $>=0$. It should be recalled that in the discussion of the de Rham cohomology operators and the Hodge decomposition theorem, one says that the definition of the harmonic form $h$ $(\Delta h=0)$ implies that $h$ is closed $(d h=0)$ and co-closed $(\delta h=0)$ together (see, e.g., Refs. $[9,10])$. We note that similar conclusions can be drawn here from the properties of the set of local and conserved charges $W, Q_{B}$ and $Q_{D}$ (or the set $W, Q_{A D}$ and $Q_{A B}$ ).

\section{Summary and discussion}

We have shown that the BRST invariant two-form gauge theory in four $(3+1)$ dimensions has an additional nilpotent symmetry, called the dual BRST, which keeps the gauge fixing term invariant. The anti-commutator of both the nilpotent charges (viz., $Q_{B}$ and $Q_{D}$ ) is the generator $(W)$ of a bosonic symmetry transformation, under which, the ghost terms remain invariant. We can see the parallel between the BRST and the dual-BRST symmetry: The nilpotent (anti)BRST symmetry transformations leave the kinetic energy term (more precisely the curvature term) of the free Abelian 2-form gauge theory invariant. On the other hand, it is the gauge-fixing term that remains invariant under the (anti)dual BRST symmetry transformations. Another parallel is: Like the BRST invariant Lagrangian density (3.1) can be written as the sum of kinetic energy- and the BRST exact terms i.e, $\mathcal{L}_{K E}+\frac{1}{\eta} \delta_{B}(F)$ (where $F$ is a function of the local fields), in the same way, we can also

express (3.1) as the sum of gauge-fixing and the co-BRST exact parts i.e, $\mathcal{L}_{G F}+\frac{1}{\eta} \delta_{D}(G)$, namely;

$$
\begin{aligned}
& \mathcal{L}_{D}=B_{\nu}\left(\partial_{\mu} B^{\mu \nu}-\partial^{\nu} \phi_{1}\right)+\frac{1}{\eta} \delta_{D}(G) \\
& G=\frac{1}{2} C_{\mu} \mathcal{B}^{\mu}-\frac{1}{6} \varepsilon_{\mu \nu \lambda \sigma} C^{\mu} H^{\nu \lambda \sigma}-\left(\partial^{\mu} C_{\mu}+\lambda\right) \phi_{2}-\left(\partial_{\mu} \bar{C}^{\mu}+\rho\right) \beta
\end{aligned}
$$

We have exploited the above symmetries to construct a field theoretical model for the Hodge theory on the four dimensional Minkowskian manifold where all the de Rham cohomology operators $(d, \delta, \Delta)$ have their counterparts as the conserved and nilpotent charges (corresponding to $d$ and $\delta$ ) and the bosonic conserved charge $W$ (corresponding to the Laplacian operator $\Delta$ ) for the BRST invariant version of the free 2-form Abelian gauge theory. All these charges are local and they generate the symmetry transformations for the Lagrangian density of this theory. In the framework of the BRST formalism, it turns out that the analogue of the Laplacian operator (i.e., $W$ ) can be represented in two different ways $\left(W=\left\{Q_{B}, Q_{D}\right\}=\left\{Q_{A B}, Q_{A D}\right\}\right)$. Thus, $d$ and $\delta$ have two representations (i.e., $\left.d \equiv Q_{B}, Q_{A D}, \delta \equiv Q_{D}, Q_{A B}\right)$ in terms of the nilpotent charges.

The bosonic symmetry generator W (anticommutator of $Q_{B}$ and $Q_{D}$ ) turns out to be the Casimir operator for the extended BRST algebra. Under the transformation, generated 
by the Casimir operator, all the fermionic fields either do not transform or transform by a vector gauge transformation (e.g., $\delta_{W} C_{\mu}=i \kappa \partial_{\mu} \lambda, \delta_{W} \bar{C}_{\mu}=-i \kappa \partial_{\mu} \rho$ ). It will also be noticed that all the gauge-fixing terms, for the bosonic as well as the fermionic fields, transform to the equations of motion under this transformations (cf. eqn. (4.1)). There exists a discrete symmetry transformation in the theory (cf. eqns. (2.7) and (3.7)) which behaves like the analogue of the Hodge $*$ operation. In fact, it relates the nilpotent transformations $\delta_{D}$ and $\delta_{B}$ in a similar fashion as there exists a relationship between the dual-exterior derivative $\delta$ $(\delta= \pm * d *)$ and the exterior derivative $d$ in differential geometry.

We summarise the main results : (i) We have found out a possible mapping between the de Rahm cohomology operators of differential geometry and the symmetry generators of a $3+1$ dimensional field theoretical model for the Hodge theory. (ii) We have shown the existence of a mapping between Hodge * operation and the discrete transformations on the fields of the theory. Both these mappings can be concisely expressed as

$$
\begin{array}{lll}
\text { Exterior derivative } d & \Leftrightarrow & Q_{B}, Q_{A D} \\
\text { Co- exterior derivative } \delta & \Leftrightarrow Q_{D}, Q_{A B} \\
\text { Laplacian } \Delta & \Leftrightarrow W=\left\{Q_{B}, Q_{D}\right\}=\left\{Q_{A B}, Q_{A D}\right\} \\
\text { Hodge * operation } & \Leftrightarrow \text { symmetry transformations }(2.7) \text { and (3.7). }
\end{array}
$$

(iii) The constraints, emerging from $Q_{B} \mid$ phys $>=0$ and $Q_{D} \mid$ phys $>=0$, are related to each-other due to the existence of the discrete duality transformations (3.7) for the bosonic part of the Lagrangian density (3.1). (iv) We see that the Lapalcian operator $W$ does not go to zero on-shell. This property was claimed to be one of the salient features of the topological field theory in Refs. [18-20] where the topological nature of the free 2D (non)Abelian 1-form gauge theory was established. Furthermore, we are unable to express the Lagrangian density (3.1) as the sum of BRST- and dual BRST invariant parts. This, in turn, implies that the energy-momentum tensor can also be not expressed as the sum of BRST- and dual BRST anticommutators. In addition, we are unable to obtain the topological invariants of the theory under consideration. Thus, 2-form free Abelian gauge theory in 4D does not mimic all the features of the free 2D 1-form gauge theory as a field theoretical model for the Hodge theory.

It will be interesting to explore the possibility of extending our investigations to the case of interacting (non)Abelian two-form gauge theory where matter fields are also present. The existence of new symmetries and their generalizations might turn out to be useful in the context of the proof for the renormalizability of such theories. It would be useful if we could discuss the $B \wedge F$ (non)Abelian gauge theory, in the framework of BRST cohomology and Hodge decomposition theorem where the 1-form gauge fields and 2-form gauge fields are coupled to each-other in a topologically invariant way. Its further extension to include matter fields is another workable problem. These are some of the issues which are under investigations and our results would be reported in our future publications. 


\section{Acknowledgements}

One of us (EH) would like to thank the Director, SNBNCBS, Calcutta for the warm hospitality extended to him during his visit to the Centre where a part of this work was done. He also thanks U.G.C., Govt. of India, for the financial support through S.R.F. scheme. Fruitful conversations with A. Lahiri are gratefully acknowledged. EH and MS thank V. Srinivasan for encouragement.

\section{References}

[1] Becchi C Rouet A and Stora R 1974 Phys. Lett. B 32344

Becchi C Rouet A and Stora R 1975 Commun. Math. Phys. 42127

Becchi C Rouet A and Stora R 1976 Ann. Phys. (N.Y.) 98287

[2] Tyutin I V 1975 Lebedev Institute Report No. Preprint FIAN-39

[3] Dirac P A M 1964 Lectures on Quantum Mechanics (New York: Yeshiva University Press)

[4] Sundermeyer K 1982 Constrained Dynamics (Lecture Notes in Physics) (Berlin: Springer)

[5] Gitman D M and Tyutin I V 1990 Qauntization of Fields with Constraints (Berlin: Springer)

[6] Nishijima K 1986 Progress in Quantum Field Theory eds. Ezawa H and Kamefuchi S (Amsterdam: North-Holland) pp. 99

[7] Henneaux M and Teitelboim C 1992 Quantization of Gauge Systems (Princeton: Princeton University Press)

[8] Nakanishi N and Ojima I 1990 Covariant Operator Formalism of Gauge Theories and Quantum Gravity (Singapore: World Scientific)

[9] Eguchi T Gilkey P B and Hanson A J 1980 Phys. Rep. 66213

[10] Mukhi S and Mukunda N 1990 Introduction to Topology, Differential Geometry and Group Theory for Physicists (New Delhi: Wiley Eastren)

[11] Nishijima K 1988 Prog. Theor. Phys. 80897 and 905

Fülöp G and Marnelius R 1995 Nucl. Phys. B 456442

Kalau W and van Holten J W 1991 Nucl. Phys. B 361233

[12] van Holten J W 1990 Phys. Rev. Lett. 642863

van Holten J W 1990 Nucl. Phys. B 339158

Aratyn H 1990 J. Math. Phys. 311240

[13] McMullan D and Lavelle M 1993 Phys. Rev. Lett. 713758

McMullan D and Lavelle M 1995 Phys. Rev. Lett. 754151

[14] Rivelles V O 1995 Phys. Rev. Lett. 754150

Rivelles V O 1996 Phys. Rev. D 533257 
[15] Marnelius R 1997 Nucl. Phys. B 494346

[16] Yang H S and Lee B -H 1996 J. Math. Phys. 376106

[17] Zhong T and Finkelstein D 1994 Phys. Rev. Lett. 733055

Zhong T and Finkelstein D 1995 Phys. Rev. Lett. 754152

[18] Malik R P 2000 Int. J. Mod. Phys. A 151685

[19] Malik R P 2000 J. Phys. A: Math. Gen. 332437

[20] Malik R P 1999 Mod. Phys. Lett. A 141937

[21] Malik R P New local symmetry for QED Preprint hep-th/0003128

[22] Seiberg N and Witten E 1999 J. High Energy Phys. (JHEP) 09032 hep-th/9908142

[23] Kalb M and Ramond P 1974 Phys. Rev. D 92273

[24] Vilenkin A and Vachaspati T 1987 Phys. Rev. D 351138

[25] Davis R L 1989 Phys. Rev. D 404033

Davis R L 1990 Mod. Phys. Lett. A 5955

[26] Allen T J Bowick M J and Lahiri A 1989 Phys. Lett. B 23747

[27] Harikumar E and Sivakumar M 1998 Phys. Rev. D 573794

[28] Lahiri A 1997 Mod. Phys. Lett. A 122699

[29] Kaul R K 1978 Phys. Rev. D 181127

[30] Lahiri A 1993 Mod. Phys. Lett. A 82403

[31] Banerjee R and Barcelos-Neto J 1998 Ann. Phys. (N. Y.) 265134

Banerjee R 1999 J. Phys. A: Math. Gen. 32517

[32] Harikumar E and Sivakumar M 2000 Nucl. Phys. B 565385

[33] Lahiri A 1997 Phys. Rev. D 555045

[34] Ogievetsky V I and Polubarinov I V 1967 Sov. J. Nucl. Phys. (Iad. Fiz.) 4156

[35] Deser S 1969 Phys. Rev. 1871931

[36] Chryssomalakos C de Azcarraga J A Macfarlane A J and Perez Bueno J C 1999 J. Math. Phys. 406009

[37] Malik R P and Pradhan T 1985 Z. Phys. C 28525

[38] Banerjee R and Chakraborty B 1999 J. Phys. A: Math. Gen. 324441 Banerjee R and Wotzasek C 1998 Nucl. Phys. B 527402 